Correspondence Hiroaki Ohnishi onishi@ks.kyorin-u.ac.jp

\section{Mycobacterium kyorinense sp. nov., a novel, slow-growing species, related to Mycobacterium celatum, isolated from human clinical specimens}

Mitsuhiro Okazaki, ${ }^{1}$ Kiyofumi Ohkusu, ${ }^{2}$ Hiroyuki Hata, ${ }^{2}$ Hiroaki Ohnishi, ${ }^{1}$ Keiko Sugahara, ${ }^{3}$ Chizuko Kawamura, ${ }^{4}$ Nagatoshi Fujiwara, ${ }^{5}$ Sohkichi Matsumoto, ${ }^{5}$ Yukiko Nishiuchi, ${ }^{6}$ Kouichi Toyoda, ${ }^{7}$ Hajime Saito, ${ }^{8}$ Shota Yonetani, ${ }^{1}$ Yoko Fukugawa, ${ }^{1}$ Masayuki Yamamoto, ${ }^{9}$ Hiroo Wada, ${ }^{9}$ Akiko Sejimo, ${ }^{3}$ Akio Ebina, ${ }^{4}$ Hajime Goto, ${ }^{9}$ Takayuki Ezaki ${ }^{2}$ and Takashi Watanabe ${ }^{1}$

\footnotetext{
${ }^{1}$ Department of Laboratory Medicine, Kyorin University School of Medicine, 6-20-2 Shinkawa, Mitaka-shi, Tokyo 181-8611, Japan

${ }^{2}$ Department of Microbiology, Regeneration and Advanced Medical Science, Gifu University Graduate School of Medicine, Gifu, 1-1 Yanagido, Gifu City, Gifu 501-1194, Japan

${ }^{3}$ National Hospital Organization Tokyo National Hospital, 3-1-1 Takeoka, Kiyose-shi, Tokyo 204-0023, Japan

${ }^{4}$ Central Laboratory, Aomori Prefectural Hospital, 2-1-1 Higashizoudou, Aomori-shi, Aomori 030-8553, Japan

${ }^{5}$ Department of Host Defense, Osaka City University, Graduate School of Medicine, 1-4-3 Asahi-machi, Abeno-ku, Osaka-shi 545-8585, Japan

${ }^{6}$ Toneyama Institute for Tuberculosis Research, Osaka City University Medical School, Osaka, Japan

${ }^{7}$ Kyokuto Pharmaceutical Industrial Co. Ltd, 7-8, Nihonbashi-kobunachou, Chuuou-ku, Tokyo, 103-0024, Japan

${ }^{8}$ Hiroshima Environment \& Health Association, Health Science Center, 9-1 Hirosekita-machi, Naka-ku, Hiroshima 730-8631, Japan

${ }^{9}$ Department of Respiratory Medicine, Kyorin University School of Medicine, 6-20-2 Shinkawa, Mitaka-shi, Tokyo 181-8611, Japan
}

\begin{abstract}
A novel, non-pigmented, slow-growing mycobacterium was identified on the basis of biochemical and nucleic acid analyses, as well as growth characteristics. Three isolates were cultured from clinical samples (two from sputum and one from pus in lymph nodes) obtained from three immunocompetent patients with infections. Bacterial growth occurred at $28-42{ }^{\circ} \mathrm{C}$ on Middlebrook 7H11-OADC agar. The isolates showed negative results for Tween hydrolysis, nitrate reductase, semiquantitative catalase, urease activity, 3 day arylsulfatase activity, pyrazinamidase, tellurite reduction and niacin accumulation tests, but positive results for 14 day arylsulfatase activity and heat-stable catalase tests. The isolates contained $\alpha-$, keto-, and dicarboxymycolates in their cell walls. Sequence analysis revealed that all isolates had identical, unique $16 \mathrm{~S}$ rRNA sequences. Phylogenetic analysis of the $16 \mathrm{~S}$ rRNA, rpoB, hsp65 and sodA gene sequences confirmed that these isolates are unique but closely related to Mycobacterium celatum. DNA-DNA hybridization of the isolates demonstrated less than $50 \%$ reassociation with M. celatum and Mycobacterium branderi. On the basis of these findings, a novel species designated Mycobacterium kyorinense sp. nov. is proposed. The type strain is KUM $060204^{\top}$ $\left(=\mathrm{JCM} 15038^{\top}=\right.$ DSM $\left.45166^{\top}\right)$.
\end{abstract}




\section{INTRODUCTION}

The advent of new molecular techniques has evoked great interest in the identification and classification of nontuberculous mycobacteria that cause infectious diseases in mammals. Currently, there are more than 100 species of nontuberculous mycobacteria, of which approximately 60 are considered to be potential pathogens (Brown-Elliott \& Wallace, 2005). Among the non-tuberculous mycobacteria, Mycobacterium celatum was first described by Butler et al. (1993). It has unique species-specific 16S rRNA and superoxide dismutase sequences, resembling those of Mycobacterium xenopi, but is biochemically indistinguishable from the Mycobacterium avium complex (Butler et al., 1993). Sequencing analysis of the $16 \mathrm{~S}$ rRNA gene in additional $M$. celatum strains revealed the existence of a new subtype, which is distinct from but very similar to the two previously reported subtypes (types 1 and 2) (Butler et al., 1993). Furthermore, Mycobacterium branderi was described as a novel species in 1995, and analysis of its $16 \mathrm{~S}$ rRNA sequence has confirmed the close phylogenetic relationship of this organism to M. celatum (Koukila-Kahkola et al., 1995).

In this study, slow-growing mycobacteria with a close phylogenetic relationship to M. celatum were isolated from clinical specimens from three Japanese patients with infections. Biochemical tests and genotypic analyses revealed that these isolates belong to the same novel species of the genus Mycobacterium for which the name Mycobacterium kyorinense is proposed.

\section{METHODS}

Bacterial strains. Strain KUM $060204^{\mathrm{T}}$ was isolated from the sputum of a 62-year-old Japanese man with pneumonia at Kyorin University Hospital in Mitaka City, Tokyo, Japan. Three more isolates were obtained from the sputa of the same patient at different time points, but later analyses revealed that all four isolates were identical. Therefore, these four isolates were referred to as a single strain, KUM $060204^{\mathrm{T}}$. Strain NTH 512-121 was isolated from the sputum of a 70-year-old Japanese man with pneumonia at the National Hospital Organization Tokyo National Hospital in Kiyose City, Tokyo, Japan. Strain AHM 060905 was isolated from pus from cervical lymph nodes of a 64-yearold Japanese woman with non-tuberculous mycobacterial lymphadenitis at Aomori Prefectural Hospital in Aomori City, Aomori, Japan. None of these three patients suffered underlying immunocompromising disease. Since strains KUM $060204^{\mathrm{T}}$ and AHM 060905 fulfil the criteria for infections of clinical significance (Medical Section of the American Lung Association, 1997), these two strains were considered to be clinically relevant in immunocompetent patients. M. celatum ATCC $51131^{\mathrm{T}}$, M. branderi ATCC $51789^{\mathrm{T}}$ and M. branderi ATCC 51788 were purchased from ATCC and used as standard strains.

Phenotypic properties. Bacterial morphology and acid-alcoholfastness were determined by Ziehl-Neelsen staining as described by Chapin (2007). Colony morphology, pigmentation, and the ability of the isolates to grow at various temperatures $\left(25,28,30,35\right.$ and $\left.42{ }^{\circ} \mathrm{C}\right)$ were observed on Middlebrook 7H11-OADC agar (Nippon Becton Dickinson) and 1\% Ogawa egg agar (Kyokuto Pharmaceutical Industrial Co. Ltd). The following biochemical tests were performed as described by Kent \& Kubica (1985): Tween hydrolysis, nitrate reductase, pyrazinamidase, tellurite reduction, urease activity, niacin accumulation, arylsulfatase activity (3- and 14-day), semiquantitative catalase and heat-stable catalase tests $\left(68^{\circ} \mathrm{C}\right)$.

Antimicrobial susceptibility testing. MICs for amikacin, clarithromycin, ethambutol, isoniazid, kanamycin, levofloxacin, rifampicin and streptomycin were determined based on the broth microdilution method with Broth MIC NTM (Kyokuto Pharmaceutical Industrial Co. Ltd) as described in monograph M24-A of the National Committee on Clinical Laboratory Standards, now CLSI (NCCLS, 2003).

Mycolic acid analysis by thin-layer chromatography (TLC). Mycolic acid analyses were performed using Silica gel TLC (Uniplate, $20 \times 20 \mathrm{~cm}, 250 \mu \mathrm{m}$; Analtech) and matrix-assisted laser desorption/ ionization time-of-flight mass spectrometry (MALDI-TOF MS) with an Ultraflex II (Bruker Daltonics) as described previously (Masaki et al., 2006) with some modifications. Mycobacterium tuberculosis H37Rv ATCC $27294^{\mathrm{T}}$, M. avium ATCC $25291^{\mathrm{T}}$ and Mycobacterium intracellulare ATCC $13950^{\mathrm{T}}$ were used as controls.

Sequence determination and phylogenetic analysis. DNA was prepared after mechanical disruption of bacterial cells and subjected to sequence analysis of the $16 \mathrm{~S}$ rRNA, $h s p 65, r p o B$ and sodA genes using methods as described by Kirschner et al. (1993), Telenti et al. (1993), Boor et al. (1995) and Domenech et al. (1997) with some modifications. Phylogenetic trees with bootstrap values were generated using the CLUSTAL $\mathrm{W}$ program (www.clustal.org) and displayed using TREEVIEW as described by $\mathrm{Li}$ et al. (2004). Phylogenetic analyses were also performed using the neighbourjoining method with Kimura's two-parameter distance correction model with 100 bootstrap replications in the MEGA version 2.1 software package. Homology searching with the 16S rRNA, hsp65, $r p o B$ and sodA gene sequences was performed against sequences registered in GenBank/EMBL/DDBJ using BLAST.

DNA-DNA hybridization. Quantitative microplate DNA-DNA hybridization for selected strains was carried out under optimal conditions as described by Ezaki et al. $(1988,1989)$.

\section{RESULTS AND DISCUSSION}

The three isolates examined were acid-alcohol-fast, nonmotile, non-spore-forming bacilli. Mature colonies of all isolates developed in 4 weeks on Middlebrook 7H11-OADC agar. Growth was observed at temperatures in the range 28$42{ }^{\circ} \mathrm{C}$, with optimal growth obtained at $30-35{ }^{\circ} \mathrm{C}$. No growth was observed at $25{ }^{\circ} \mathrm{C}$. Colonies were smooth and initially transparent, but became creamy white on prolonged culture.

All three isolates were negative for Tween hydrolysis, nitrate reductase, semiquantitative catalase, urease activity, 3-day arylsulfatase activity, pyrazinamidase, tellurite reduction and niacin accumulation, but positive for 14-day arylsulfatase activity and heat-stable catalase (Table 1). All isolates were distinguishable from $M$. celatum and $M$. branderi by a lack of growth at $25{ }^{\circ} \mathrm{C}$ and a negative pyrazinamidase test. They were also distinguishable from $M$. celatum by a negative result for 3-day arylsulfatase activity and the tellurite reduction test. The three isolates were also distinct from Mycobacterium cookii by their ability to grow at $45{ }^{\circ} \mathrm{C}$ and a lack of growth at $25^{\circ} \mathrm{C}$, absence of pigment production in the dark, and a negative semiquantitative catalase test. They were also distinct from 
Table 1. Growth characteristics of strain KUM $060204^{\top}$ in comparison with the closely related species $M$. celatum and $M$. branderi

+, Positive; -, negative; ND, not done.

\begin{tabular}{|lccc|}
\hline Characteristic & $\begin{array}{c}\text { KUM } \\
\mathbf{0 6 0 2 0 4}^{\mathbf{T}}\end{array}$ & $\begin{array}{c}\text { M. } \\
\text { celatum }^{*}\end{array}$ & $\begin{array}{c}\text { M. } \\
\text { branderi }^{*}\end{array}$ \\
\hline Growth temperature $\left({ }^{\circ} \mathrm{C}\right)$ & & & \\
25 & - & + & + \\
28 & + & + & $\mathrm{ND}$ \\
30 & + & $+\left(31{ }^{\circ} \mathrm{C}\right)$ & $\mathrm{ND}$ \\
35 & + & $\mathrm{ND}$ & $\mathrm{ND}$ \\
42 & + & $+\left(45^{\circ} \mathrm{C}\right)$ & $+\left(45^{\circ} \mathrm{C}\right)$ \\
Heat-stable catalase & + & + & - \\
$\left(68{ }^{\circ} \mathrm{C}\right)$ & & & \\
Arylsulfatase activity & & & \\
3 days & - & + & - \\
14 days & + & + & + \\
Pyrazinamidase & - & + & + \\
Tellurite reduction & - & + & $\mathrm{ND}$ \\
\hline
\end{tabular}

${ }^{*}$ Data taken from Butler et al. (1993), Koukila-Kahkola et al. (1995) and Vincent \& Gutierres (2007).

M. xenopi by the lack of pigment production in the dark (Koukila-Kahkola et al., 1995).

Using antimicrobial susceptibility tests, all isolates were shown to be susceptible to clarithromycin and ethambutol, but resistant to isoniazid and rifampicin. Amikacin, kanamycin, levofloxacin and streptomycin showed low MIC values against all three isolates (Table 2).

Mycolic acid analyses of the three isolates by TLC produced identical multispot patterns composed of $\alpha$ - (C82-86)-, keto (C84-89)- and dicarboxy- (C64-69) mycolates. The length of the carbon chain of each mycolic acid methyl ester was assigned by the mass number and proposed structures as summarized in Table 3. The mycolic acids contained predominantly one or two cyclopropane rings. This pattern of mycolic acid subclasses is characteristic for

Table 2. Susceptibility of the three isolates to various antibiotics

\begin{tabular}{|lccc|}
\hline \multirow{2}{*}{ Antibiotic } & \multicolumn{3}{c|}{ MIC $\left(\boldsymbol{\mu g ~ \mathbf { ~ m l } ^ { - \mathbf { 1 } } )}\right.$} \\
\cline { 2 - 4 } & KUM 060204 $^{\mathrm{T}}$ & $\mathbf{N T H ~ 5 1 2 - 1 2 1}$ & AHM 060905 \\
\hline Amikacin & $<0.5$ & $<0.5$ & $<0.5$ \\
Clarithromycin & $<0.03$ & $<0.03$ & $<0.03$ \\
Ethambutol & 4 & 2 & 4 \\
Isoniazid & 16 & 32 & 16 \\
Kanamycin & 0.5 & 0.25 & 1 \\
Levofloxacin & 0.125 & $<0.03$ & 0.125 \\
Rifampicin & 32 & $>32$ & $>32$ \\
Streptomycin & 0.25 & 0.25 & 0.25 \\
\hline
\end{tabular}

the Mycobacterium avium complex, and is also seen in $M$. celatum and $M$. branderi, suggesting that the new isolates are closely related to these Mycobacterium species (Fig. 1) (Butler et al., 1993; Brander et al., 1992).

The sequence of the 16S rRNA gene was identical in all strains, but was different from all other available $16 \mathrm{~S}$ rRNA sequences. The sequences of the $h s p 65, r p o B$ and sodA genes were also identical in the three isolates. It was assumed that these three isolates belong to the same species, and strain KUM $060204^{\mathrm{T}}$ was used as a representative strain for genotypic analyses thereafter.

Sequence analysis revealed that the 16S rRNA gene sequence of the newly identified mycobacterium was closest to that of M. celatum ATCC 51130 (type 2) with 18 base mismatches out of $1469 \mathrm{bp}$ ( $98.8 \%$ identity). It was also highly similar to that of M. celatum NCTC 12882 (type 3) with 25 base mismatches out of $1476 \mathrm{bp}$ (98.3\%), M. celatum ATCC $51131^{\mathrm{T}}$ (type 1) with 27 base mismatches out of $1450 \mathrm{bp}$ (98.1\%), M. branderi ATCC $51789^{\mathrm{T}}$ with 32 base mismatches out of $1476 \mathrm{bp}(97.8 \%)$ and M. cookii ATCC $49103^{\mathrm{T}}$ with 41 base mismatches out of $1477 \mathrm{bp}(97.2 \%)$. A phylogenetic tree was created based on the 16S rRNA gene sequences, incorporating all previously described species of slow-growing mycobacteria. Phylogenetic analysis revealed that strain KUM $060204^{\mathrm{T}}$ is adjacent to the type strains of $M$. celatum (ATCC $51131^{\mathrm{T}}$ ), M. branderi (ATCC $51789^{\mathrm{T}}$ ) and M. cookii (ATCC $49103^{\mathrm{T}}$ ) (Fig. 2a).

The hsp65 gene sequence of strain KUM $060204^{\mathrm{T}}$ was identical to that of M. celatum ATCC 51130 (392/392, $100 \%$ ) and highly similar to those of $M$. branderi ATCC $51789^{\mathrm{T}}$ and M. celatum ATCC $51131^{\mathrm{T}}$ (98.8 and $98.1 \%$, respectively). A phylogenetic tree based on the $h s p 65$ sequences is shown in Fig. 2(b). Phylogenetic analysis of the $h s p 65$ gene gave consistent results, i.e. strain KUM $060204^{\mathrm{T}}$ is located adjacent to the type strains of $M$. branderi (ATCC $51789^{\mathrm{T}}$ ) and M. celatum (ATCC $51131^{\mathrm{T}}$ ).

The sequence of the $r p o B$ gene of strain KUM $060204^{\mathrm{T}}$ was identical to that of M. celatum ATCC 51130 (306/306, 100\%) and highly similar to that of M. branderi ATCC $51789^{\mathrm{T}}$ $(96.7 \%)$. It showed further differences from those of $M$. celatum ATCC $51131^{\mathrm{T}}$ and M. cookii ATCC $49103^{\mathrm{T}}(94.1$ and $92.7 \%$, respectively). Strain KUM $060204^{\mathrm{T}}$ clustered adjacent to M. branderi ATCC $51789^{\mathrm{T}}$ and M. celatum ATCC $51131^{\mathrm{T}}$ (Fig. 2c) in a phylogenetic tree based on $r p o B$ sequences.

The sodA gene of strain KUM $060204^{\mathrm{T}}$ was almost identical to that of M. celatum ATCC $51130(411 / 413,99.5 \%)$ and highly similar to that of $M$. celatum ATCC $51131^{\mathrm{T}}$ $(96.6 \%)$. It showed further differences from those of $M$. branderi ATCC $51789^{\mathrm{T}}$ and M. xenopi ATCC $19250^{\mathrm{T}}(90.1$ and $86.4 \%$, respectively) (Fig. $2 \mathrm{~d}$ ).

Given the close relationship of strain KUM $060204^{\mathrm{T}}$ to $M$. celatum and M. branderi, a DNA-DNA hybridization study was performed for these strains under optimal conditions (Table 4). Strain KUM $060204^{\mathrm{T}}$ exhibited DNA similarity values below the suggested species threshold $(70 \%)$ to its 
Table 3. The relationship between molecular species and mass numbers in each mycolic acid subclass

Underlining indicates a major component.

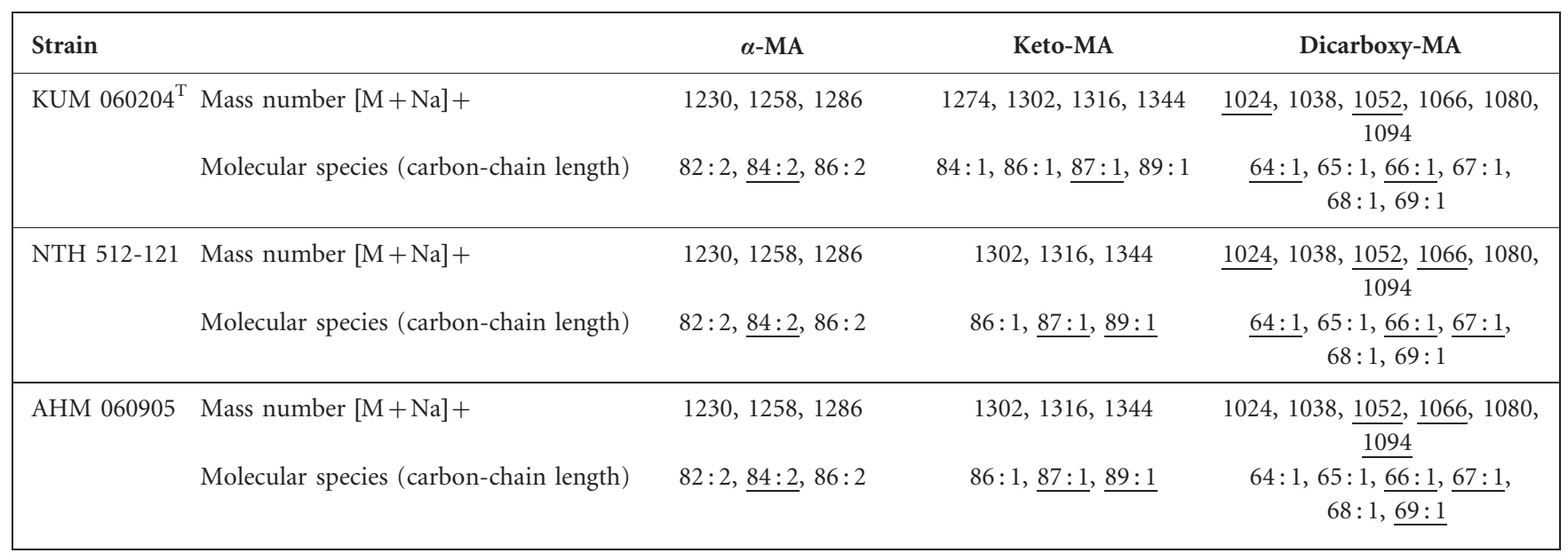

phylogenetic neighbours M. celatum ATCC $51131^{\mathrm{T}}, M$. branderi ATCC $51789^{\mathrm{T}}$ and $M$. branderi ATCC 51788 (similarity $49.9,44.5$ and $41.5 \%$, respectively). These results provide further evidence for the genetic diversity between this strain and closely related species, indicating that this isolate comprises a new mycobacterial species.

Based on the genotypic and phenotypic data described above, it was concluded that strain KUM $060204^{\mathrm{T}}$ represents a novel Mycobacterium species for which we propose the name Mycobacterium kyorinense sp. nov. Two other strains, NTH 512-121 and AHM 060905, were assumed to belong to the same species as strain KUM $060204^{\mathrm{T}}$ based on sequence identity. Considering that strains KUM $060204^{\mathrm{T}}$ and AHM 060905 fulfil the criteria for clinical significance, this newly identified Mycobacterium is considered to be a potential pathogen for infection in humans.

\section{Description of Mycobacterium kyorinense sp. nov.}

Mycobacterium kyorinense (kyo.rin.en'se. N.L. neut. adj. kyorinense of Kyorin, referring to the Kyorin University Hospital where the first strain was isolated).

Long, rod-shaped cells (approx. $3 \times 0.3 \mu \mathrm{m}$ ); acid-alcoholfast. Growth requires $>4$ weeks at $28-42{ }^{\circ} \mathrm{C}$. No growth occurs at $25^{\circ} \mathrm{C}$. Colony diameters are $1-2 \mathrm{~mm}$ on Middlebrook 7H11-OADC agar. Colonies on $1 \%$ Ogawa egg agar are smooth and raised with round or lobate regular margins, and non-chromogenic. Negative for Tween hydrolysis, nitrate reductase, semiquantitative catalase, urease activity, 3-day arylsulfatase activity, pyrazinamidase, tellurite reduction and niacin accumulation; positive for 14-day arylsulfatase activity and heat-stable catalase tests. Susceptible to clarithromycin and ethambu- (a)

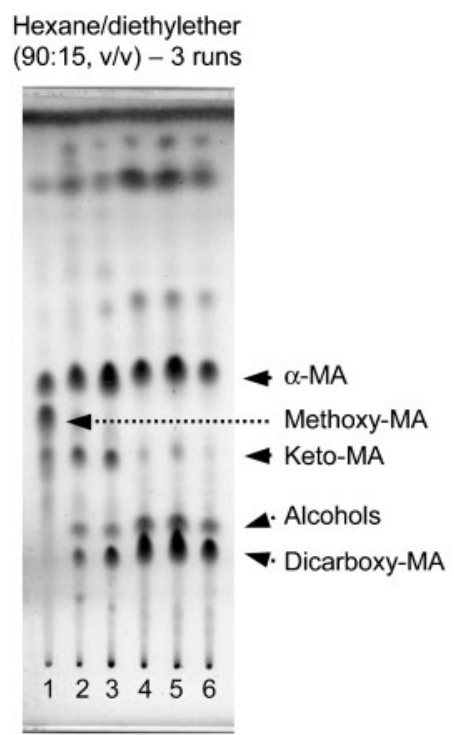

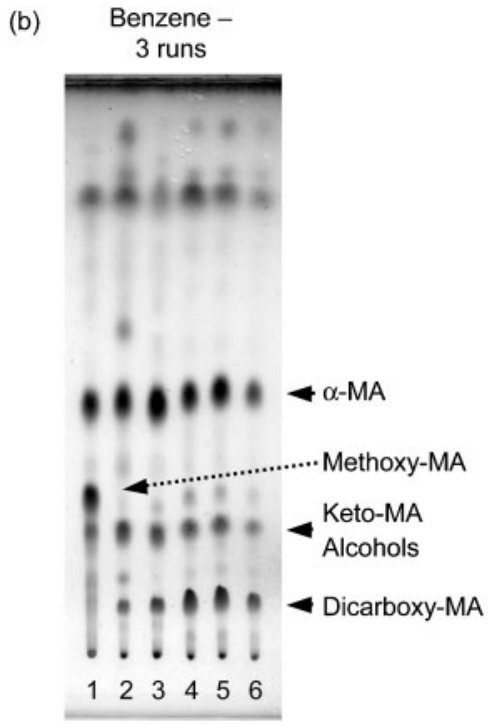

Fig. 1. TLC patterns of mycolic acid methyl esters. (a) Plate developed three times with hexane/diethylether. (b) Plate developed three times with benzene. Lanes: $1, M$. tuberculosis H37Rv ATCC $27294^{\top} ; 2$, M. avium ATCC $25291^{\top} ; 3$, M. intracellulare ATCC $13950^{\top} ; 4$, strain KUM 060204 ${ }^{\top}$; 5, strain NTH 512-121; 6 , strain AHM 060905. MA, Mycolic acid. 
(a) $16 \mathrm{~S}$ rRNA

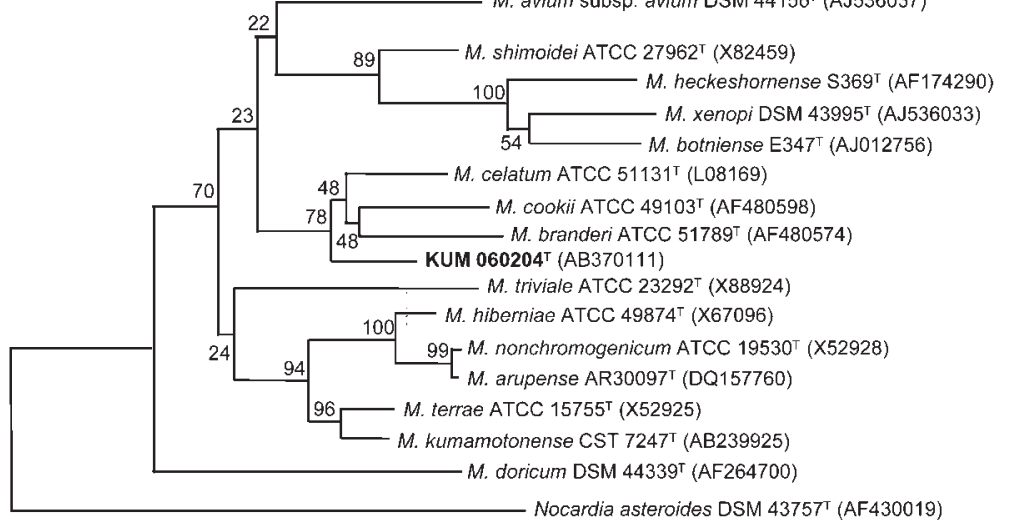

(c) $r p o B$

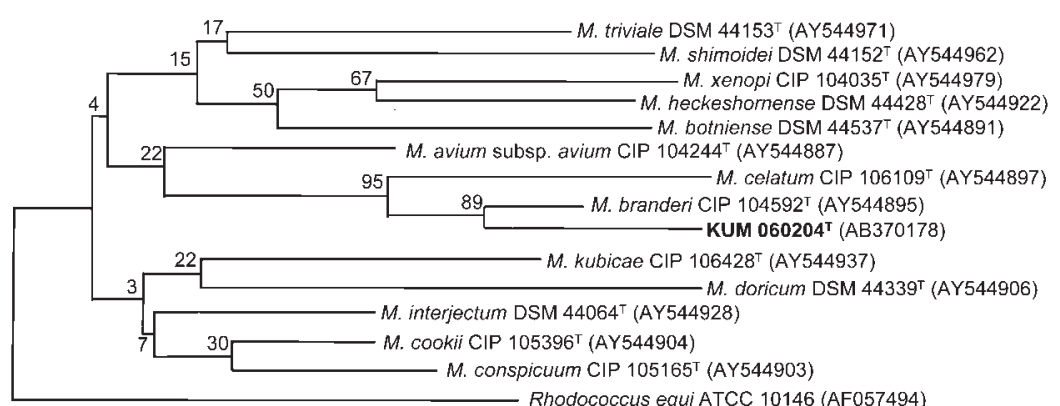

0.01 (b) $h s p 65$

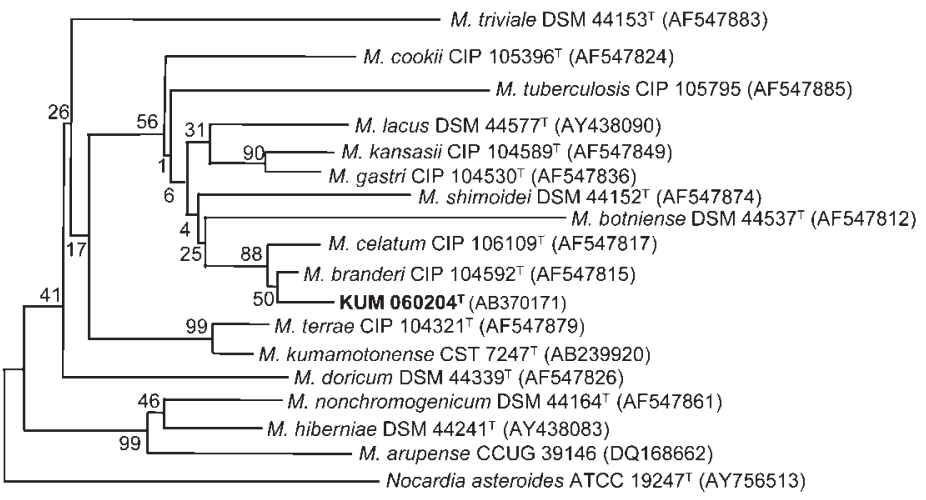

$\stackrel{0.01}{\longmapsto}$

(d) $\operatorname{sod} A$

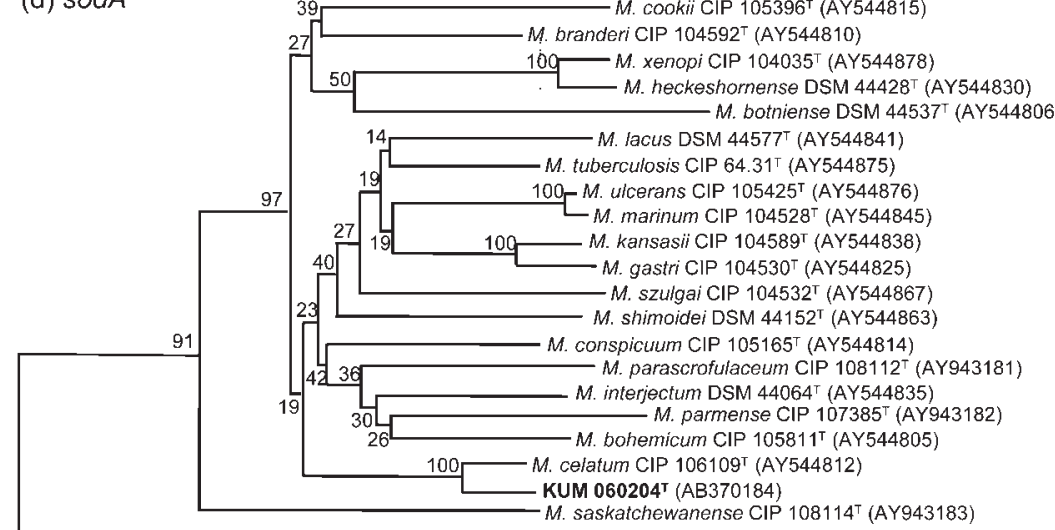

Nocardia abscessus DSM 44432T (AY544981)

0.01

Fig. 2. Phylogenetic trees for isolate KUM $060204^{\top}$ and closely related type strains of other mycobacterial species based upon comparisons of (a) $16 \mathrm{~S}$ rRNA, (b) hsp65, (c) rpoB and (d) sodA gene sequences using the neighbour-joining method. The trees were rooted using the sequences of Nocardia asteroides (16S rRNA and hsp65), Rhodococcus equi (rpoB) and Nocardia abscessus (sodA) as the outgroups. The scale bars represent a $1 \%$ sequence difference. 
Table 4. Levels of DNA-DNA relatedness between strain KUM $060204^{\top}$ and related Mycobacterium species

\begin{tabular}{|c|c|c|c|c|}
\hline \multirow[t]{2}{*}{ Strain } & \multicolumn{4}{|c|}{ Similarity value (\%) with labelled DNA from: } \\
\hline & KUM $060204^{\mathrm{T}}$ & $\begin{array}{c}\text { M. celatum ATCC } \\
51131^{\mathrm{T}}\end{array}$ & $\begin{array}{c}\text { M. branderi ATCC } \\
51789^{\mathrm{T}}\end{array}$ & $\begin{array}{c}\text { M. branderi ATCC } \\
51788\end{array}$ \\
\hline KUM $060204^{\mathrm{T}}$ & 100 & 49.1 & 46.0 & 47.6 \\
\hline M. celatum ATCC $51131^{\mathrm{T}}$ & 49.9 & 100 & 62.4 & 59.8 \\
\hline M. branderi ATCC $51789^{\mathrm{T}}$ & 44.5 & 51.3 & 100 & 100 \\
\hline
\end{tabular}

tol, but resistant to isoniazid and rifampicin. Sequence analysis of the 16S rRNA, hsp65, rpoB and $\operatorname{sodA}$ genes indicated that $M$. kyorinense is a mycobacterial species most closely related to $M$. celatum and $M$. branderi. DNA-DNA hybridization revealed that $M$. kyorinense exhibits DNA similarity values below the suggested threshold with its phylogenetic neighbours $M$. celatum ATCC $51131^{\mathrm{T}}, M$. branderi ATCC $51789^{\mathrm{T}}$ and $M$. branderi ATCC 51788 (similarity $49.9,44.5$ and $41.5 \%$, respectively), thus defining it as a distinct species. M. kyorinense was placed in the slow-growing mycobacteria group.

The type strain is KUM $060204^{\mathrm{T}}\left(=\mathrm{JCM} 15038^{\mathrm{T}}=\mathrm{DSM}\right.$ $45166^{\mathrm{T}}$ ), isolated from sputum in a patient with pneumonia.

\section{ACKNOWLEDGEMENTS}

This work was supported by grants from the Ministry of Health, Labour and Welfare (Research on Emerging and Re-emerging Infectious Diseases, Health Sciences Research Grants) of Japan, The Japan Health Sciences Foundation, Ministry of Education Culture Sports Science and Technology, and The United States-Japan Cooperative Medical Science Program against Tuberculosis and Leprosy.

\section{REFERENCES}

Boor, K. J., Duncan, M. L. \& Price, C. W. (1995). Genetic and transcriptional organization of the region encoding the beta subunit of Bacillus subtilis RNA polymerase. J Biol Chem 270, 20329-20336.

Brander, E., Jantzen, E., Huttunen, R., Julkunen, A. \& Katila, M. L. (1992). Characterization of a distinct group of slowly growing mycobacteria by biochemical tests and lipid analyses. J Clin Microbiol 30, 1972-1975.

Brown-Elliott, B. \& Wallace, R. J., Jr (2005). Infections caused by nontuberculous mycobacteria. In Principles and Practice of Infectious Diseases, vol. 2, pp. 2909-2915. Edited by G. L. Mandell, J. E. Bennett \& R. Dolin. Pennsylvania: Elsevier.

Butler, W. R., O'Connor, S. P., Yakrus, M. A., Smithwick, R. W. Plikaytis, B. B., Moss, C. W., Floyd, M. M., Woodley, C. L., Kilburn, J. O. \& other authors (1993). Mycobacterium celatum sp. nov. Int J Syst Bacteriol 43, 539-548.

Chapin, K. C. (2007). Principles of stains and media. In Manual of Clinical Microbiology, 9th edn, pp. 182-191. Edited by P. R. Murray, E. J. Baron, M. L. Landry, J. H. Jorgensen \& M. A. Pfaller. Washington, DC: American Society for Microbiology.

Domenech, P., Jimenez, M. S., Menendez, M. C., Bull, T. J., Samper, S., Manrique, A. \& Garcia, M. J. (1997). Mycobacterium mageritense sp. nov. Int J Syst Bacteriol 47, 535-540.
Ezaki, T., Hashimoto, Y., Takeuchi, N., Yamamoto, H., Liu, S. L., Miura, H., Matsui, K. \& Yabuuchi, E. (1988). Simple genetic method to identify viridans group streptococci by colorimetric dot hybridization and fluorometric hybridization in microdilution wells. $J$ Clin Microbiol 26, 1708-1713.

Ezaki, T., Hashimoto, Y. \& Yabuuchi, E. (1989). Fluorometric deoxyribonucleic acid-deoxyribonucleic acid hybridization in microdilution wells as an alternative to membrane filter hybridization in which radioisotopes are used to determine genetic relatedness among bacterial strains. Int J Syst Evol Microbiol 39, 224-229.

Kent, P. T. \& Kubica, G. P. (1985). Public Health Mycobacteriology: a Guide for the Level III Laboratory. Atlanta: US Department of Health and Human Services, Centers for Disease Control.

Kirschner, P., Springer, B., Vogel, U., Meier, A., Wrede, A., Kiekenbeck, M., Bange, F. C. \& Bottger, E. C. (1993). Genotypic identification of mycobacteria by nucleic acid sequence determination: report of a 2-year experience in a clinical laboratory. J Clin Microbiol 31, 2882-2889.

Koukila-Kahkola, P., Springer, B., Bottger, E. C., Paulin, L., Jantzen, E. \& Katila, M. L. (1995). Mycobacterium branderi sp. nov., a new potential human pathogen. Int J Syst Bacteriol 45, 549-553.

Li, Y., Kawamura, Y., Fujiwara, N., Naka, T., Liu, H., Huang, X., Kobayashi, K. \& Ezaki, T. (2004). Sphingomonas yabuuchiae sp. nov. and Brevundimonas nasdae sp. nov., isolated from the Russian space laboratory Mir. Int J Syst Evol Microbiol 54, 819-825.

Masaki, T., Ohkusu, K., Hata, H., Fujiwara, N., lihara, H., YamadaNoda, M., Nhung, P. P., Hayashi, M., Asano, Y. \& other authors (2006). Mycobacterium kumamotonense sp. nov. recovered from clinical specimen and the first isolation report of Mycobacterium arupense in Japan: novel slowly growing, nonchromogenic clinical isolates related to Mycobacterium terrae complex. Microbiol Immunol 50, 889-897.

Medical Section of the American Lung Association (1997). Diagnosis and treatment of disease caused by nontuberculous mycobacteria. This official statement of the American Thoracic Society was approved by the Board of Directors, March 1997. Am J Respir Crit Care Med 156, S1-S25.

NCCLS (2003). Susceptibility Testing of Mycobacteria, Nocardiae, and Other Aerobic Actinomycetes; Approved Standard. NCCLS document M24-A. Wayne, PA: National Committee for Clinical Laboratory Standards.

Telenti, A., Marchesi, F., Balz, M., Bally, F., Bottger, E. C. \& Bodmer, T. (1993). Rapid identification of mycobacteria to the species level by polymerase chain reaction and restriction enzyme analysis. J Clin Microbiol 31, 175-178.

Vincent, V. \& Gutierres, M. C. (2007). Mycobacterium: laboratory characteristics of slowly growing mycobacteria. In Manual of Clinical Microbiology, 9th edn, pp. 573-588. Edited by P. R. Murray, E. J. Baron, M. L. Landry, J. H. Jorgensen \& M. A. Pfaller. Washington, DC: American Society for Microbiology. 